\title{
Minimum Parent-Offspring Recombination Haplotype Inference in Pedigrees
}

\author{
Qiangfeng Zhang ${ }^{1}$, Francis Y.L. Chin ${ }^{2}$, and Hong Shen ${ }^{3}$ \\ 1 Department of Computer Science, \\ University of Science and Technology of China, Hefei 230026, China \\ qfzhang@mail.ustc.edu.cn \\ 2 Department of Computer Science, The University of Hong Kong, \\ Pokfulam, Hong Kong \\ chin@cs.hku.hk \\ 3 Graduate School of Information Science, JAIST, Ishikawa, Japan \\ shen@jaist.ac.jp
}

\begin{abstract}
The problem of haplotype inference under the Mendelian law of inheritance on pedigree genotype data is studied. The minimum recombination principle states that genetic recombinations are rare and haplotypes with fewer recombinations are more likely to exist. Given genotype data on a pedigree, the problem of Minimum Recombination Haplotype Inference (MRHI) is to find a set of haplotype configurations consistent with the genotype data having the minimum number of recombinations. In this paper, we focus on a variation of the MRHI problem that gives more realistic solutions, namely the $k$-MRHI problem, which has the additional constraint that the number of recombinations in each parent-offspring pair is at most $k$. Although the $k$-MRHI problem is NPhard even for $k=1$, the $k$-MRHI problem with $k>1$ can be solved efficiently by dynamic programming in $O\left(n m_{0}^{3 k+1} 2^{m_{0}}\right)$ time by adopting an approach similar to the one used by Doi, Li and Jiang [4] on pedigrees with $n$ nodes and at most $m_{0}$ heterozygous loci in each node. In particular, the 1-MRHI problem can be solved in $O\left(n m_{0}^{4} 2^{m_{0}}\right)$ time. We propose an $O\left(n^{2} m_{0}\right)$ algorithm to find a node as the root of the pedigree tree so as to further reduce the time complexity to $O\left(m_{0} \min \left(t_{R}\right)\right)$, where $t_{R}$ is the number of feasible haplotype configuration combinations in all trios in the pedigree tree when $R$ is the root. If the pedigree has few generations, the 1-MRHI problem can be solved in $O\left(\min \left\{n m_{0}^{4} 2^{m_{0}}, n m_{0}^{l+1} 2^{\mu_{R}+l}\right\}\right)$ time, where $\mu_{R}$ is the number of heterozygous loci in the root, and 1 is the maximum path length from the root to the leaves in the pedigree tree. Experiments on both real and simulated data confirm the out-performance of our algorithm when compared with other popular algorithms. In most real cases, our algorithm gives the same haplotyping results but runs much faster. In some real cases, other algorithms give an answer which has the least number of recombinations, while our algorithm gives a more credible solution and runs faster.
\end{abstract}

\section{Introduction}

The modeling of human genetic variation is critical to the understanding of the genetic basis for complex diseases. Single nucleotide polymorphisms (SNPs [13] ) 
are the most frequent form of this variation. The Human Genome Project and other large-scale efforts have identified millions of SNP markers that can be used in genetic studies. Although each marker can be analyzed independently, it is much more informative to analyze them in groups. Therefore, it is useful to analyze haplotypes (haploid genotypes), which are sequences of linked markers on a single chromosome. In diploid organisms, such as humans, chromosomes come in pairs, and experiments often yield genotype information, which blend haplotype information for chromosome pairs. There is growing evidence that, in order to better characterize the role of a candidate gene, full haplotype information should be exploited instead of using only genotype information. Unfortunately, it is both time-consuming and expensive to derive haplotype information experimentally. This explains the increasing interest in inferring haplotype information, or haplotyping, computationally [2] [.

Input genotype data can be with or without any other pedigree information. Haplotyping pedigree data is believed to be more reliable than haplotyping population data for unrelated individuals: the constraint provided by parents-offspring relationships in a pedigree could force one to settle on a unique haplotype configuration as being most probable.

Genetic research shows that recombinations are rare in human data [5]. The genomic DNA can be partitioned into long blocks such that recombinations within each block are rare or even nonexistent. Thus it is believed that haplotype configurations with fewer recombinations should be preferred in haplotype inference [11] 12].

The Minimum-Recombination Haplotype Inference (MRHI) problem, which is NP-hard [4, is to find a haplotype configuration with minimum number of recombinations for a given pedigree genotype data. Various algorithms have been presented for the MRHI problem [8] [7] 12] [15]. In some cases, however, the MRHI model might yield unrealistic results in which a few parent-offspring pairs have many recombinations while others have no or few recombinations. We present a more realistic problem, called the $k$-MRHI problem which basically is the MRHI problem, but with an additional constraint that the number of recombinations in each parent-offspring pair is bounded by a constant $k$. The $k$-MRHI problem is NP-hard even for $k=1$.

The $k$-MRHI problem can be solved by a dynamic programming (DP) algorithm which is very similar to the algorithm by Doi, Li and Jiang [4]. By avoiding studying all $2^{3 m_{0}}$ haplotype configurations in each parents-offspring trio, our algorithm takes $O\left(n m_{0}^{4} 2^{m_{0}}\right)$ time when $k=1$, instead of the $O\left(n m_{0} 2^{3 m_{0}}\right)$ time needed by [4 for the MRHI problem on pedigrees with $n$ nodes and at most $m_{0}$ heterozygous loci in each node. Note that not all nodes have $m_{0}$ heterozygous loci, and the number of feasible haplotype configurations at a node is limited by the number of feasible haplotype configurations of its neighbors, and thus the number of possible haplotype configurations at a node can be much less than $2^{m_{0}}$. This observation leads to the idea of choosing different nodes in the pedigree as the root of the tree in speeding up the algorithm. The main contributions of this paper are: (1) to define a more realistic problem for haplotype inference 
( $k$-MRHI), (2) to give a more efficient and practical DP algorithm for the haplotype inference problem with improved time complexities, and (3) to present an efficient algorithm to find the root in the pedigree for better performance in the DP algorithm.

\section{Preliminaries}

Haplotypes and genotypes consist of linked genetic markers which are small segments of DNA with some specific features. The physical position of a marker on a chromosome is called a locus and its state is called an allele. Without loss of generality, the two alleles of a biallelic (2-state) SNP can be denoted by ' 0 ' and ' 1 ', and a haplotype $h$ with $m$ loci is presented as a string of length $m$ over $\{0,1\}^{m}$, and a genotype $g$ as a string over $\{0,1,2\}^{m}$. Haplotype pair $\left\langle h_{1}, h_{2}\right\rangle$ is compatible with a genotype $g$ if (a) the two alleles of $h_{1}$ and $h_{2}$ are the same at the same locus, for example ' 0 ' (respectively ' 1 '), then the corresponding locus of $g$ should also be ' 0 ' (respectively ' 1 '), which denotes a homozygous site; otherwise, (b) the two alleles of $h_{1}$ and $h_{2}$ are different, then the corresponding site of $g$ should be '2', which denotes a heterozygous site.

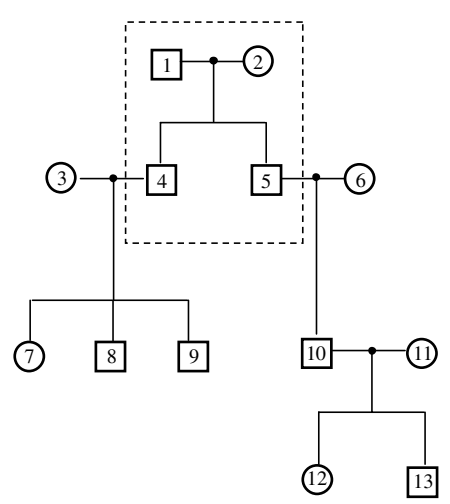

(a)

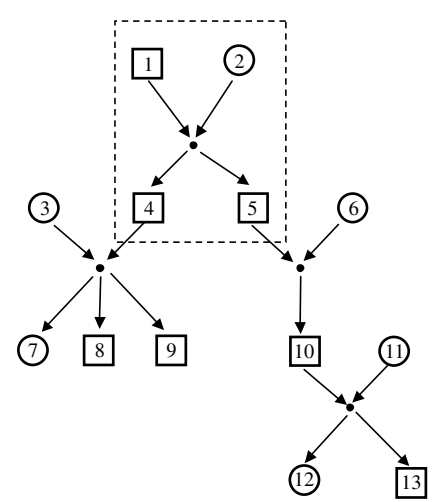

(b)

Fig. 1. The pictorial representation and graph representation of a pedigree

A pedigree is a fundamental structure used in genetics. Figure 1(a) shows the pictorial representation (used by the biologists) of a pedigree with 13 nodes. A square represents a male node, a circle represents a female node, and a black dot represents a mating node. The subgraph in the dashed square is a typical nuclear family, which contains a father (node 1), a mother (node 2) and two children (nodes 4 and 5 ). The children are placed under their parents. Nodes 1 , 2 and 4 consist a parents-offspring trio, nodes 1 and 4 , nodes 1 and 5 , nodes 2 
and 4 , nodes 2 and 5 are parent-offspring pairs. We define a pedigree formally as in [4].

Defintion 14. A pedigree is a weakly connected directed acyclic graph $P=$ $(V, E)$, where $V=M \cup F \cup N$, with $M$ stands for the male nodes, $F$ the female nodes, $N$ the mating nodes, and $E=\{(u, v)\}$ with $u \in M \cup F$ and $v \in N$, alternatively $u \in N$ and $v \in M \cup F$.

Figure 1(b) shows the graph representation of the pedigree given in Figure 1(a). A sub-graph containing the father, the mother, and their children is a nuclear family. A nuclear family can also be represented by a mating node which connects them together. A parents-offspring trio, or just trio, consists of two parents and one of their children; and a parent-offspring pair (PO-pair) refers to a father and his child or a mother and her child. In this paper, we assume that the pedigree never forms a cycle if the directions of edges are ignored (no mating-loop).

Each individual node in a pedigree is associated with its genotype. In the absence of genetic mutation, at each locus, the child must inherit one allele from its father and the other from its mother. This is known as the Mendelian law of inheritance. Usually, one haplotype of a child is inherited as a whole from one of the two haplotypes of a parent. However, recombinations may occur, where the two haplotypes of a parent get shuffled due to a crossover of a chromosome and one of the shuffled copies (recombinant) is passed on to the child. However, genetic research shows that recombinations are rare in human genetics. Thus we are interested in finding the haplotype configurations such that the total number of recombinations in the whole pedigree is minimized.

Defintion 2[12. Minimum Recombinant Haplotype Inference (MRHI) Problem: Given a pedigree graph $P$, each individual node of $P$ associates with a genotype. Find a haplotype configuration for the pedigree that each haplotype pair at each node is an explanation of its corresponding genoytype and the total number of recombinations is minimized.

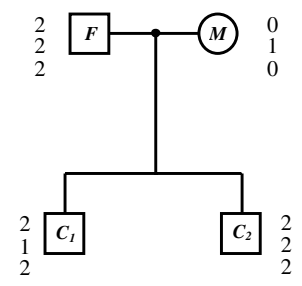

(a)

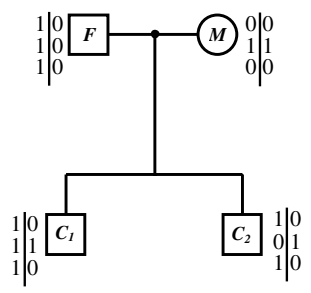

(b)

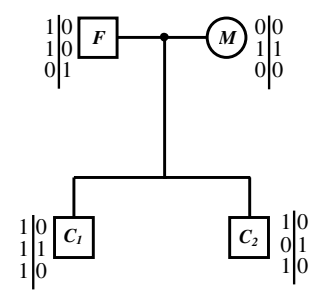

(c)

Fig. 2. Two different solutions for a pedigree of a nuclear family

Figure 2(a) shows a pedigree of a nuclear family containing a father $F$, a mother $M$ and 2 children $C_{1}, C_{2}$. Figure $2(\mathrm{~b})$ gives a solution with no recom- 
bination in trio $\left(F, M, C_{1}\right)$ and 2 recombinations in trio $\left(F, M, C_{2}\right)$. Figure $2(\mathrm{c})$ gives another solution, which also has 2 recombinations in total, but at most one recombination in each trio, i.e. at most one recombination in each PO-pair. As genetic studies show that recombinations are rare to have 2 recombinations within one PO-pair, e.g., there are $13 \%$ single recombinations versus $0.84 \%$ double recombinations in the Drosophila autosomal genes [5, Figure 2(c) should be a more credible solution than Figure 2(b) for the haplotype inference problem.

Defintion 3 . $k$-Recombination Haplotype Inference ( $k$-MRHI) Problem: Given a pedigree graph $P$ with each individual node associated with a genotype, find a haplotype configuration that is compatible with the genotypes at all nodes having the minimum number of recombinations and no more than $k$ recombinations in each PO-pair.

\section{A Dynamic Programming Algorithm for $k$-MRHI}

\subsection{The 1-MRHI Problem $(k=1)$}

In 4, Doi et al. gives a proof for the NP-hardness of the MRHI problem by a reduction from MAX CUT. In their construction, the number of recombinations within each PO-pair is limited to 1 . This trivially implies that the $k$-MRHI problem, even for $k=1$, is also NP-hard.

However, in most cases, we can find a feasible solution for a $k$-MRHI instance with $k<2$. As we have mentioned before, more than 1 recombination within a PO-pair is very unlikely in reality. Therefore, we shall focus on the 1-MRHI problem first and generalize to the $k$-MRHI problem later.

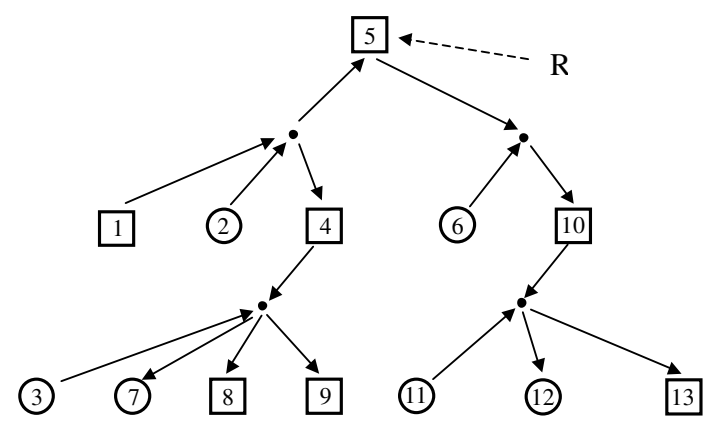

Fig. 3. The searching tree of the pedigree in Figure 1

A locus-based dynamic programming (DP) algorithm for the $k$-MRHI problem was presented in [4, with a time complexity of $O\left(n m_{0} 2^{3 m_{0}}\right)$, where $m_{0}$ is the maximum number of heterozygous loci in the genotype at each node of a loopless pedigree. We adopt a similar DP approach to solve the 1-MRHI problem 
by (1) assigning an arbitrary node $R$ in the pedigree as the root (an example is given in Figure 3, which shows a rooted tree at node 5 for the pedigree in Figure $1)$; (2) recursively finding num $[R][s]$, the minimum number of recombinations required for all feasible haplotype configurations $s$ of $R$; and (3) selecting the haplotype with the minimum number of recombinations as the solution.

Let num $[r][s]$ denote the minimum number of recombinations required in the sub-tree rooted at $r$ with the haplotype configuration $s$ under the constraint that there is at most 1 recombination in each $\mathrm{PO}$-pair of the sub-tree. If $r$ has multiple mating nodes as its tree sons, we compute each mating node separately. Each child mating node of $r$ defines a unique nuclear family, which may contain $r$ as a parent or a child and the computation of $n u m[r][s]$ is performed recursively in that nuclear family.

Suppose that the nuclear family consists of father $F$, mother $M$ and children $C_{1}, \cdots, C_{d}$. If $r$ is a leaf node, $\operatorname{num}[r][s]=0$ for any of haplotype configuration $s$; else, if $r$ is $M$ (or $F$, respectively) with haplotype configuration $s$, then:

$$
\operatorname{num}[r][s]=\min _{p}\left(\operatorname{num}[F][p]+\sum_{i=1}^{d} \min _{c_{i}}\left(\operatorname{num}\left[C_{i}\right]\left[c_{i}\right]+\operatorname{numtrio}\left(p, s, c_{i}\right)\right)\right)
$$

where $p$ denotes the haplotype configuration at node $F$ and $c_{i}$ the haplotype configuration at $C_{i}$, one of the $d$ children in this nuclear family. numtrio $\left(p, s, c_{i}\right)$ returns the minimum number of recombinations required for a trio consisting of $F, M$, and $C_{i}$ with the haplotype configurations $p, s$ and $c_{i}$ respectively, under the constraint that no $\mathrm{PO}$-pair can have more than one recombination. If there does not have any feasible solution, then numtrio $\left(p, s, c_{i}\right)$ will return $\infty$, which indicates "no solution".

Similarly, if $r$ is $C_{j}$ with haplotype configuration $s$, then we have:

$$
\begin{aligned}
\operatorname{num}[r][s] & =\min _{p, q}(\text { numtrio }[p, q, s]+\operatorname{num}[F][p]+\text { num }[M][q] \\
& \left.+\sum_{i=1, i \neq j}^{d} \min _{c_{i}}\left(\text { num }\left[C_{i}\right]\left[c_{i}\right]+\text { numtrio }\left(p, q, c_{i}\right)\right)\right) \quad \text { where } r=C_{j}
\end{aligned}
$$

where $p, q$ and $c_{i}$ are defined as before for haplotype configurations at $F, M$ and $C_{i}$ respectively.

Note that the above algorithm is the same as that presented in [4], and thus would have the same time complexity. However, a reduction in time complexity is possible from an important observation: it is not necessary to consider all combinations of haplotype configurations in each trio, which number $O\left(2^{3 m_{0}}\right)$ in total, because many combinations of haplotype configurations will be infeasible, i.e. will not have at most one recombination per $\mathrm{PO}$-pair.

For example, assume the genotype of $F$ is $(2,2, \cdots, 2)$ of length $m_{0}$ and with haplotype configuration $s=\left\langle h_{s 1}, h_{s 2}\right\rangle$ and $h_{c 1}$ in the haplotype $c=\left\langle h_{c 1}, h_{c 2}\right\rangle$ of $C_{i}$ is inherited from $s$ with no more than 1 recombination. There are $m_{0}+1$ ways of forming $h_{c 1}$ by inheriting its first $w$ alleles from the first $w$ alleles in $h_{s 1}$ and the remaining $\left(m_{0}-w\right)$ alleles from $h_{s 2}$ with $0 \leq w \leq m_{0}$. Similarly, there 
are another $m_{0}+1$ ways of forming $h_{c 1}$ from the first $w$ alleles in $h_{s 2}$ and the remaining $\left(m_{0}-w\right)$ alleles from $h_{s 1}$. Since there are double-counting in these two cases when $w=0$ and $m_{0}$, the number of feasible haplotype configurations of $c$ is limited to $2 m_{0}$, and the time complexity of the algorithm can be much reduced if we limit the number of configurations needed to be searched for the optimal result. More precisely, suppose $r$ in Equation (1) is $M$ (or $F$ ), and $s=\left\langle h_{s 1}, h_{s 2}\right\rangle$, let $N_{s}$ be the set of feasible haplotype configurations $c=\left\langle h_{c 1}, h_{c 2}\right\rangle$ that can be inherited by child $C_{i}$ from $s$ of $r$ with no more than one recombination. Thus, $\left|N_{s}\right| \leq 2 m_{0}$. As $h_{c 2}$ is inherited from the haplotype configuration $q=\left\langle h_{q 1}, h_{q 2}\right\rangle$ of $F$, let $N_{c}$ be the set of feasible haplotype configurations of $F$ which can produce the haplotype configuration $c$ in $C$ with no more than one recombination. Let $N_{s, C_{i}}^{\prime}=\cup_{c \in N_{s}} N_{c}$, which indicates the set of feasible haplotype configurations at $F$ which can go together with haplotype $s$ at $M$ to produce children $C_{i}$ with no more than one recombination in the father-child pair and in the mother-child pair. Obviously, $N_{s, C_{i}}^{\prime} \leq 4 m_{0}^{2}$.

As each haplotype configuration of $F$ should be able to produce any of the children $C_{1}, \cdots, C_{d}$, the set of feasible haplotype configurations in $F$ is $N_{s}^{\prime}=$ $\cap_{i} N_{s, C_{i}}^{\prime}$. Equation (1) can be rewritten as:

$$
\operatorname{num}[r][s]=\min _{p \in N_{s}^{\prime}}\left(n u m[F][p]+\sum_{i} \min _{c_{i} \in N_{s}}\left(n u m\left[C_{i}\right]\left[c_{i}\right]+\operatorname{numtrio}\left(p, s, c_{i}\right)\right)\right)
$$

As for Equation (2), if $r$ is $C_{j}$ and its haplotype configuration $s=\left\langle h_{s 1}, h_{s 2}\right\rangle$, let $N_{s, F}$ and $N_{s, M}$ be the sets of feasible haplotype configurations in $F$ and $M$, which can produce $C_{j}$ with haplotype configuration $s$. As $\left|N_{s, F}\right| \leq 2 m_{0}$ and $\left|N_{s, M}\right| \leq 2 m_{0}$, let $N_{p, C_{i}}\left(N_{q, C_{i}}\right)$ be the set of feasible haplotype configurations on another child $C_{i}$ with haplotype configuration $p$ in $F(q$ in $M)$ and $N_{p, q}^{\prime \prime}=$ $N_{p, C_{i}} \cap N_{q, C_{i}}$ be the set of feasible haplotype configurations for each child $C_{i}$ which can concurrently appear with the haplotype configuration $s$ of child $C_{j}$. Note that $N_{p, q}^{\prime \prime} \leq 2 m_{0}$ and Equation (2) can be rewritten as:

$$
\begin{aligned}
\operatorname{num}[r][s] & =\min _{p \in N_{s, F}, q \in N_{s, M}}(\text { numtrio }(p, q, s)+\text { num }[F][p]+\text { num }[M][q] \\
& \left.+\sum_{i \neq j} \min _{c_{i} \in N_{p, q}^{\prime \prime}}\left(\text { num }\left[C_{i}\right]\left[c_{i}\right]+\text { numtrio }\left(p, q, c_{i}\right)\right)\right) \quad \text { where } r=C_{j}
\end{aligned}
$$

Theorem 1. The above dynamic programming algorithm can solve the 1-MRHI problem in $O\left(n m_{0}^{4} 2^{m_{0}}\right)$ time and $O\left(n 2^{m_{0}}\right)$ space for pedigree with $n$ nodes and at most $m_{0}$ heterozygous loci in each node.

Proof. The rooted tree can be constructed in $O(n)$ time. As we have to consider the $8 m_{0}^{3}$ combinations in each trio for each haplotype configuration of a node and we need $O\left(m_{0}\right)$ time to compute numtrio for each haplotype configuration combination in a trio, it may take $O\left(m_{0}^{4} 2^{m_{0}}\right)$ time to process each trio. There are at most $n$ parent-offspring trios in the pedigree, so the time complexity is $O\left(n m_{0}^{4} 2^{m_{0}}\right)$. Furthermore, we need to store the array num and pointers for backtracking. The size of num is $O\left(n 2^{m_{0}}\right)$, so is the number of pointers. Thus the space complexity is $O\left(n 2^{m_{0}}\right)$. 


\subsection{The $k$-MRHI Problem}

We have argued that in most cases, feasible solutions exist for 1-MRHI. However, there are still some instances that require more recombinations within each POpair. In almost all practical cases, there are at most 2 recombinations within each PO-pair. In the following, we generalize the DP algorithm to the general $k$-MRHI problem with some modifications.

We need to modify the definition of neighboring haplotype configurations set from $N_{s}$ to $N_{s}^{(k)}$ : for each haplotype configuration $c=\left\langle h_{c 1}, h_{c 2}\right\rangle \in N_{s}^{(k)}$, one of $\left\langle h_{c 1}, h_{c 2}\right\rangle$ is inherited from one of $\left\langle h_{s 1}, h_{s 2}\right\rangle$ with no more than $k$ recombinations. So we have $\left|N_{s}^{(k)}\right|=O\left(m_{0}^{k}\right)$.

Similarly, we modify the definition of $N_{s}^{\prime(k)}=\cap_{i} N_{s, C_{i}}^{\prime(k)}$ with $N_{s, C_{i}}^{\prime}$ to $N_{s, C_{i}}^{\prime(k)}$ in Equation (3) and the definition of $N_{s, F}$ and $N_{s, M}$ to $N_{s, F}^{(k)}$ and $N_{s, M}^{(k)}, N_{p, C_{i}}$ to $N_{p, C_{i}}^{(k)}$, and $N_{p, q}^{\prime \prime}$ to $N_{p, q}^{\prime \prime}(k)$ in Equation (4). Then we have:

Theorem 2. The time complexity of the DP algorithm solving the $k$-MRHI problem is $O\left(\mathrm{~nm}_{0}^{3 k+1} 2^{m_{0}}\right)$ for pedigree with $n$ nodes and at most $m_{0}$ heterozygous loci in each node.

\section{Root Selection for Better Performance}

We have shown in Section 3 that in the 1-MRHI problem, the number of feasible haplotype configuration combinations in each trio is no more than $O\left(m_{0}^{2} 2^{m_{0}}\right)$. However, in practice the feasible haplotype configuration combinations in each trio may be much less than that because of the following reasons: (1) not all nodes have $m_{0}$ heterozygous loci; and (2) the number of feasible haplotype configurations $a_{v}$ of a node $v$ is also bounded by the number of feasible haplotype configurations $a_{v_{r}}$ of $v$ 's neighbor $v_{r}$, which can participate in the feasible haplotype configuration combinations in a trio, i.e., $a_{v} \leq 2 \mu_{v} a_{v_{r}}$, where $\mu_{v}$ is the number of heterozygous loci in $v$. Thus, different selections of a node in the pedigree as the root for the DP algorithm will give different processing times. The following we shall discuss an algorithm to find the best root based on the estimated number of feasible haplotype configurations in each node.

Starting from any node $R$, as root and assuming $\alpha_{R}$ be the number of feasible haplotypes configurations of $R$, i.e., $\alpha_{R}=2^{\mu_{R}}$, we will traverse the tree in pre-order and, for each node $v$, evaluate the number of the feasible haplotype configurations for its neighboring nodes.

If $v$ has multiple mating nodes as $v$ 's children, we compute each mating node separately. Each mating node as $v$ 's child defines a unique nuclear family, which may contain $v$ as a parent or a child. Suppose that the nuclear family consists of father $F$, mother $M$ and children $C_{1}, \cdots, C_{k}$.

If $v$ is $M$ (or $F$, respectively), $\alpha_{C_{i}}=\min \left\{2^{\mu_{C_{i}}}, 2 \mu_{C_{i}} \alpha_{v}\right\} \quad(i=1, \cdots, k)$ and $\alpha_{F}=\min _{i}\left\{2^{\mu_{F}}, 2 \mu_{F} \alpha_{C_{i}}\right\}$. If $v$ is $C_{i}$, then $\alpha_{F}=\min \left\{2^{\mu_{F}}, 2 \mu_{F} \alpha_{v}\right\}, \alpha_{M}=$ $\min \left\{2^{\mu_{M}}, 2 \mu_{M} \alpha_{v}\right\}$ and $\alpha_{C_{i}}=\min \left\{2^{\mu_{C_{i}}}, 2 \mu_{C_{i}} \alpha_{F}, 2 \mu_{C_{i}} \alpha_{M}\right\}(i=1, \cdots, k)$. Thus, 
the number of feasible haplotype configuration combinations $t_{i}$ in trio $T_{i}$ can be computed consequently, assuming an arbitrary node (node $R$ ) as the root of the searching tree. The total number of feasible haplotype configuration combinations in all trios in the pedigree is $t_{R}=\sum_{i} t_{i}$, which can be computed by a tree traversal.

Theorem 3. Let $m_{0}$ be the number of heterozygous loci and $t_{R}$ be the total number of feasible haplotype configuration combinations for all trios in the pedigree with node $R$ as root. Then the node which gives $\min \left(t_{R}\right)$ can be found in $O\left(n^{2} m_{0}\right)$ time and the 1-MRHI problem can be solved in $O\left(m_{0} \min \left(t_{R}\right)\right)$ time.

Proof. We can evaluate $t_{R}$ for each node $R$ in the pedigree in $O\left(n m_{0}\right)$ time and choose the node with $\min \left(t_{R}\right)$ as the root in $O\left(n^{2} m_{0}\right)$ time. As the computation of numtrio for each feasible haplotype configuration combination in each trio takes $O\left(m_{0}\right)$ time, the 1-MRHI problem can be solved in $O\left(m_{0} \min \left(t_{R}\right)\right)$ time after selecting the best root for the DP algorithm.

\subsection{Special Pedigrees with Few Generations}

We notice that the diameters of the pedigree graphs in many practical instances are usually small. For example, the 452 families in the CEPH database [1] [3] [10] consist of only three generations, usually with four grandparents, two parents and a number of children. Figure 4 shows a typical family (family 1413) with 21 members. The longest path starts from one of the grandparents from the father's side to one of the grandparents from the mother's side and is of length 4 (not counting the mating nodes). But if we start from any of the children, we can reach any other node within 2 steps.

Suppose that the number of heterozygous loci in the chosen root $R$ is $\mu_{R}$, and any other nodes can be reached within $l$ steps from $R$. We shall enumerate all the $2 \mu_{R}$ feasible haplotype configurations of the root in the first step, and no more than $2^{\mu_{R}} \times 2 m_{0}$ feasible haplotype configurations for each of its neighboring nodes in the second step, and so on. The number of feasible haplotype configurations

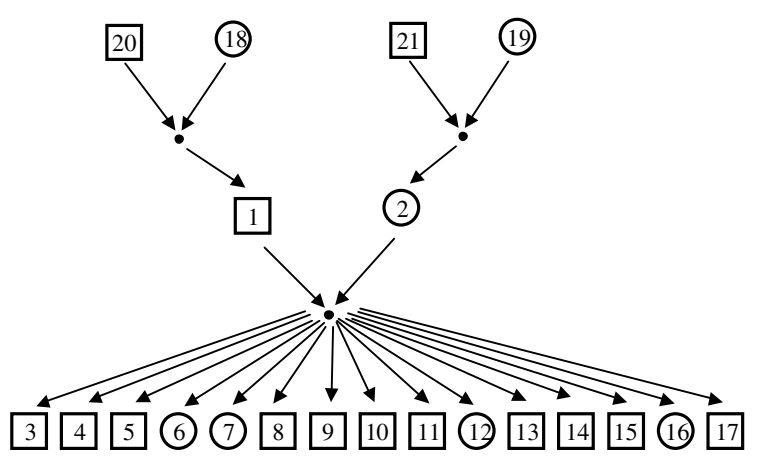

Fig. 4. A typical family(family 1413) in the CEPH database 
is at most $2^{\mu_{R}} \times\left(2 m_{0}\right)^{l}$ at the most distant node. When $\mu_{R} \ll m_{0}$ and $l$ is relatively small, we will get an improvement in the time complexity:

Theorem 4. 1-MRHI can be solved in $\min \left\{O\left(n m_{0}^{4} 2^{m_{0}}\right), O\left(n m_{0}^{l+1} 2^{\mu_{R}+l}\right)\right\}$ time for pedigree with $n$ nodes and at most $m_{0}$ heterozygous loci in each node, where $l$ is the maximum path length from the root to the leaves and $\mu_{R}$ is the number of heterozygous loci in root $R$.

Proof. We have to consider all combinations of feasible haplotype configurations at nodes in each trio, which is at most $2^{\mu_{R}} \times\left(2 m_{0}\right)^{l}$. We need $O\left(m_{0}\right)$ time to compute numtrio for each haplotype configuration combination in each trio, and there may be at most $O(n)$ trios, the time complexity of the algorithm is $\min \left\{O\left(n m_{0}^{4} 2^{m_{0}}\right), O\left(n m_{0}^{l+1} 2^{\mu_{R}+l}\right)\right\}$.

\section{$5 \quad$ Experimental Results}

We implemented the above DP algorithm in $\mathrm{C}++$, and all experiments were conducted on a Pentium IV PC with $1.7 \mathrm{GHz}$ CPU and 256MB RAM.

\subsection{Real Data}

We examined a real data set on Epsiodic Ataxia (EA) by Litt et al. [9] which involves a family containing 29 people typed at 9 polymorphic markers on chromosome $12 p$. Both the locus-based algorithm [4] and the 1-MRHI algorithm run fast $(t<1$ sec.) on this data set but the results are different. The locus-based algorithm gives a feasible solution with 5 recombinations in total but with a double recombination in one haplotype of member 100. The 1-MRHI algorithm, however, finds a more credible solution that has 6 recombinations in total, but with at most 1 recombination for each haplotype in the pedigree.

Another two real data sets are three generations families like those in the CEPH database [1] [10] ( ftp://genome.wi.mit.edu/distribution/mpg/hapmap/ hap_struct/popA/ (Gabriel et al.)): family 1331 on chromosome $7 a$, and family 1346 on chromosome 2 a. After removing the loci with missing alleles, family 1331 is a pedigree consisting of 8 members on 32 loci, and family 1346 is a pedigree consisting of 8 members on 55 loci. Both the locus-based algorithm and the 1MRHI algorithm give the same answer for family 1331 , but take $522.4 s$ and $8.7 \mathrm{~s}$, respectively. As for family 1346, the locus-based algorithm fails because of not enough resources while the 1-MRHI algorithm finds out a solution in 31 minutes.

\subsection{Simulated Data}

We compare the performance of our algorithm, with the locus-based algorithm [4] and PHASE 14, a widely used program based on Gibbs Sampling algorithm, the running time $t$ and the accuracy ratio $\rho$ (in recovering the correct haplotypes). We used three different tree pedigree structures in the experiment: (1) a tree 
with 13 nodes (Figure 1), (2) a tree with 29 nodes (Figure 8 in [7]), (3) a typical family with 21 nodes from the CEPH database [1] 3] 10] (Figure 5).

For each pedigree, genotypes with 15 and 30 biallelic marker loci are considered. The two alleles at each locus of a founder are independently sampled with a fixed frequency of 0.5 . The recombination rate is set to $r=0,0.1,0.2$ between generations, and we limit the number of recombinations to no more than one in each PO-pair. For each combination of the above parameters, 100 sets of random genotype data are generated and the average performance of the programs is computed, as shown in Table 1.

Table 1. Comparison of performances of different haplotyping programs on simulation data

\begin{tabular}{|c|c|c|c|c|c|c|c|c|}
\hline \multirow[b]{3}{*}{$(n, r)$} & \multicolumn{6}{|c|}{$m=15$} & \multirow{2}{*}{\multicolumn{2}{|c|}{$\begin{array}{l}m=30 \\
1-\mathrm{MRHI}\end{array}$}} \\
\hline & \multicolumn{2}{|c|}{ locus-based $^{[4]}$} & \multicolumn{2}{|c|}{ PHASE $^{[14]}$} & \multicolumn{2}{|c|}{ 1-MRHI } & & \\
\hline & $t(s e c)$. & $\rho$ & $t(s e c)$. & $\rho$ & $t($ sec. $)$ & $\rho$ & $t(s e c)$. & $\rho$ \\
\hline$(13,0.0)$ & 255.7 & 1.00 & 688.2 & .87 & 1.68 & 1.00 & 202.8 & 1.00 \\
\hline$(29,0.0)$ & 576.3 & 1.00 & 1772.8 & .91 & 12.33 & 1.00 & 839.6 & 1.00 \\
\hline$(21,0.0)$ & 234.4 & 1.00 & 592.4 & .95 & 1.02 & 1.00 & 44.0 & 1.00 \\
\hline$(13,0.1)$ & 287.7 & .93 & 972.3 & .85 & 1.73 & .91 & 241.1 & .92 \\
\hline$(29,0.1)$ & 542.8 & .90 & 2210.2 & .90 & 10.45 & .90 & 1042.8 & .94 \\
\hline$(21,0.1)$ & 243.2 & .91 & 1504.2 & .93 & 0.52 & .94 & 33.7 & .96 \\
\hline$(13,0.2)$ & 294.2 & .85 & 1221.4 & .85 & 3.17 & .89 & 1032.4 & .86 \\
\hline$(29,0.2)$ & 613.5 & .81 & 3022.2 & .89 & 11.70 & .84 & 916.1 & .84 \\
\hline$(21,0.2)$ & 244.1 & .90 & 2106.7 & .93 & 1.22 & .95 & 47.4 & .92 \\
\hline
\end{tabular}

$\dagger$ Average performance is obtained from 100 independent executions of each program and for each parameter setting. $n$ stands for the number of nodes, $m$ for the number of marker loci, $r$ for the recombination rate, $t(s e c$.) for the average running time, and $\rho$ for the accuracy ratio.

₹ The locus-based algorithm cannot be applied to cases of $m \geq 30$, due to the space limitation. PHASE is also excluded for cases of $m \geq 30$ because of the time.

As we can see from the table, 1-MRHI runs quickest, and the locus-based algorithm runs quicker than PHASE. Thus the 1-MRHI algorithm can be applied to much larger instances than the locus-based algorithm and PHASE can (the other two algorithms fail when $m=30$ ).

In terms of the quality of solutions, all three algorithms can recover the correct haplotype configurations with high probabilities. The accuracy ratio decreases with the increase in the number of recombinations, which is more obvious for the locus-based algorithm and the 1-MRHI algorithm. Since we have limited the number of recombinations within each PO-pair to no more than 1 in the data, the locus-based algorithm, which often finds solutions with fewer recombinations than the actual haplotype configurations, performs worse than the 1-MRHI algorithm as expected. 


\section{Concluding Remarks}

1-MRHI brings an improvement on the running time of solving the general MRHI problem, even though 1-MRHI and the general MRHI usually give the same solutions as confirmed from the experiments. If the solutions are different, 1-MRHI usually gives the more credible solutions. In some cases, if the total number of recombinations for 1-MRHI solutions is much larger than the total number of recombinations for 2-MRHI solutions, then it is plausible that the 2-MRHI solution should be more credible. Our next goal is to find the most probable haplotype configuration which can explain the genotypes in a pedigree when the probabilities of single, double, triple recombinations are given.

Our algorithm for $k$-MRHI cannot deal with mating loops; nor can the locusbased DP algorithm [4. A member-based DP algorithm [4] can deal with pedigrees with mating loops, but may not be well-suited to solving the $k$-MRHI problem because of the increase in time complexity. In practice, pedigree data often contains missing alleles. It will be interesting to find an efficient algorithm for $k$-MRHI on pedigrees with mating loop and genotypes with missing data.

Acknowledgement. We thank T. Jiang and J. Li for sharing their DP code with us and Dr. M.Y. Chan for proof-reading the first draft of this paper. Thanks to the RGC grant HKU 7135/04E for supporting this research.

\section{References}

1. The CEPH genotype database. http://www.cephb.fr/.

2. A.G. Clark. Inference of haplotypes from PCR-amplified samples of diploid populations. Mol. Biol. Evol, 7(2):111-122, 1990.

3. J. Dausset, H. Cann, D. Cohen, M. Lathrop, J.M. Lalouel, and R. White. Centre d'etude du polymorphisme humain (ceph): collaborative genetic mapping of the human genome. Genomics, 5:575-577, 1990.

4. K. Doi, J. Li, and T. Jiang. Minimum recombinant haplotype configuration on tree pedigree. In Proc. of WABI'03, pages 339-353, 2003.

5. A. Griffiths, W. Gelbart, R. Lewontin, and J. Miller. Modern Genetic Analysis: Integrating Genes and Genomes. W.H. Freeman and Company, N.Y., 2002.

6. D. Gusfield. Inference of haplotypes from samples of diploid populations: complexity and algorithms. J. Computational Biology, 8:305-323, 2001.

7. J. Li and T. Jiang. Efficient inference of haplotypes from genotypes on a pedigree. J. Bioinfo Comp Biol, 1(1), 2003.

8. Jing Li and Tao Jiang. Efficient inference of haplotypes from genotypes on a pedigree. In Proc. of RECOMB'03, pages 197-206, 2003.

9. M. Litt, P. Kramer, D. Browne, S. Gancher, E.R.P. Brunt, D. Root, et al. A gene for episodic ataxia/myokymia maps to chromosome 12p13. Am. J. Hum. Genet., 55:702-709, 1994.

10. J.C. Murray et al. A comprehensive human linkage map with centimorgan density. Science, 265:2049-2054, 1994.

11. J.R. O'Connell. Zero-recombinant haplotyping: applications to fine mapping using snps. Genet Epidemiol, 19, 2000. 
12. D. Qian and L. Beckmann. Minimum-recombinant haplotyping in pedigrees. $A m$ J Hum Genet, 70(6):1434-1445, 2002.

13. E. Russo et al. Single nucleotide polymorphism: Big pharma hedges its bets. The Scientist, 13, 1999.

14. M. Stephens, N.J. Smith, and P. Donnelly. A new statistical method for haplotype reconstruction for population data. Am. J. Hum. Genet, 68:978-989, 2001.

15. P. Tapadar, S. Ghosh, and P.P. Majumder. Haplotyping in pedigrees via a genetic algorithm. Hum Hered, 50(1):43-56, 2000. 